\section{Electrocardiographic diagnosis of acute myocardial infarction in the presence of left bundle branch block}

\author{
William J Brady, Francis Morris
}

It is often stated that the diagnosis of acute myocardial infarction (AMI) is extremely difficult if not impossible in the presence of left bundle branch block (LBBB). Likewise the electrocardiographic diagnosis of previous myocardial infarction is also difficult in this setting. The ST segment and $\mathrm{T}$ wave abnormalities associated with uncomplicated LBBB may mimic acute ischaemic changes. A wide range of electrocardiographic abnormalities have been suggested to establish the diagnosis of AMI in the presence of LBBB but no single set of criteria have been universally accepted nor found to be particularly useful.

The diagnosis of AMI in the presence of LBBB is extremely important as these patients have a greatly increased risk of complications and death. New LBBB suggests occlusion of the proximal left anterior descending artery resulting in a large area of jeopardised myocardium; new AMI in the setting of pre-existing LBBB places the patient a extreme risk of cardiogenic shock and death. It has been suggested that the outcome of patients with LBBB and AMI is significantly improved by acute reperfusion therapies, such as a thrombolytic agent or primary angioplasty. It is therefore important to be aware of the changes that may

indicate AMI when the electrocardiogram (ECG) is complicated by the presence of LBBB.

\section{Case reports \\ CASE 1}

A 54 year old woman presented to the accident and emergency department with a two hour history of substernal chest pain, similar to previous attacks of angina. The pain was associated with nausea and sweating. She had a past history of ischaemic heart disease, diabetes, and hypertension. A 12 lead ECG revealed sinus rhythm and LBBB (fig 1). The patient was treated with aspirin and opiate analgesia though her pain was not controlled. Shortly after presentation a further ECG (fig 2) was recorded which revealed ST segment elevation in leads II, III, and aVF as well as V5 and V6, suggesting an inferolateral myocardial infarction. The patient received a thrombolytic agent and made an uneventful recovery. AMI was confirmed by raised cardiac enzymes and an echo revealed inferior lateral hypokinesis.

\section{CASE 2}

A 61 year old man presented to the accident and emergency department with intermittent

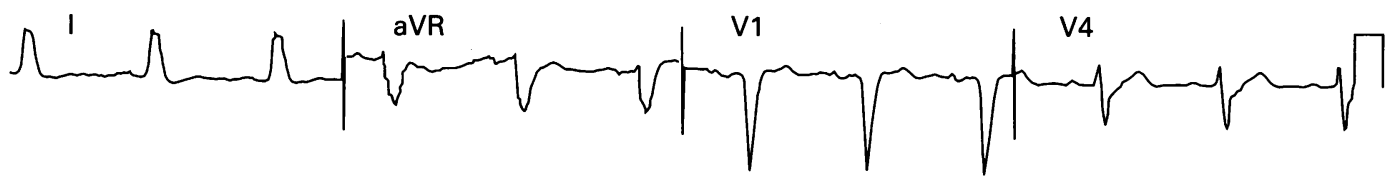

V2

V5
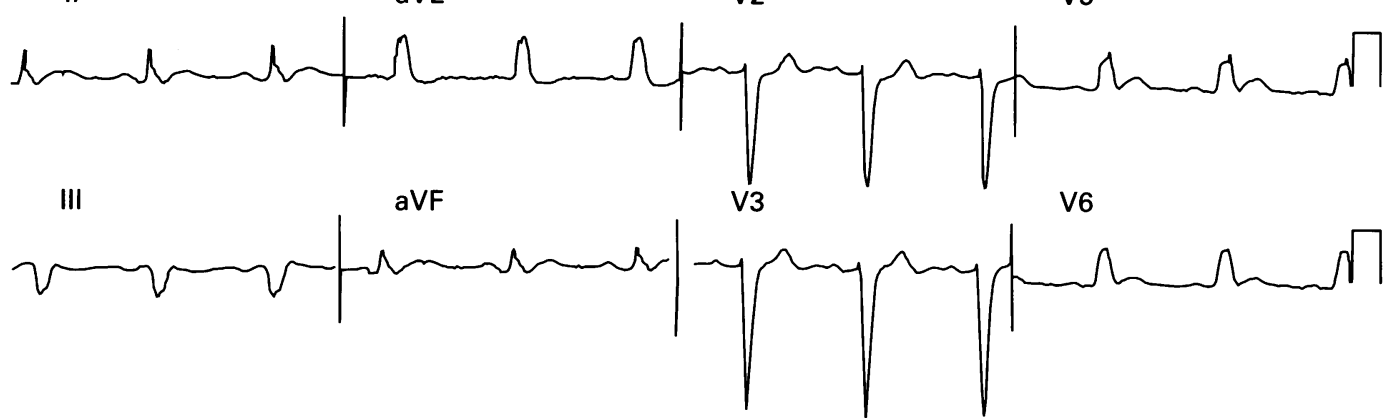

Figure 1 The ECG above reveals sinus rhythm and LBBB. A careful inspection of leads II, V5, and V6 reveals the ST segment and $T$ waves are upright and concordant (in the same direction as) with the QRS complex. The degree of ST elevation in these leads is minimal. It is important to remember that the anticipated finding in left bundle branch block involves ST segment which are discordant (in the opposite direction to) with the QRS complex. There are other, more subtle changes which are not usually seen in uncomplicated LBBB. In leads V2-V4, there is either ST segment depression (V2) or blunting of the expected ST segment elevation (V3 and V4). In LBBB, such leads with a rS configuration of $Q R S$ complex usually have marked ST elevation with tall vaulting $T$ waves. 


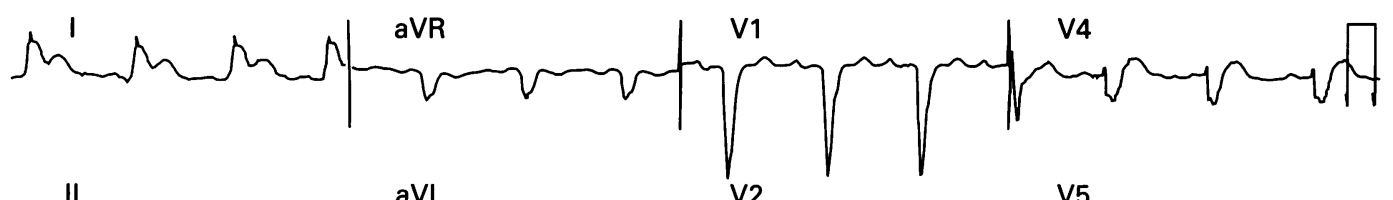

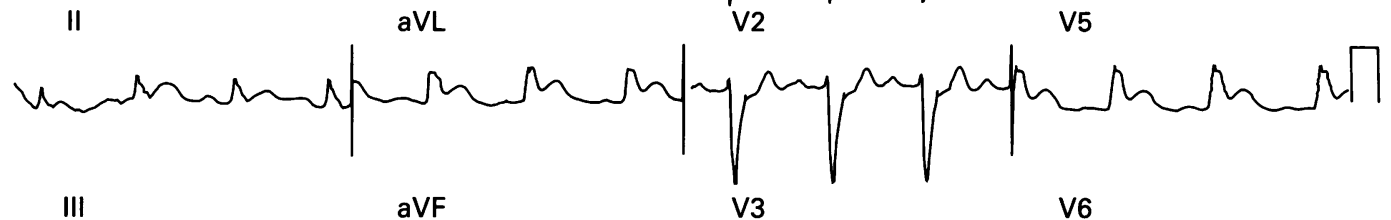

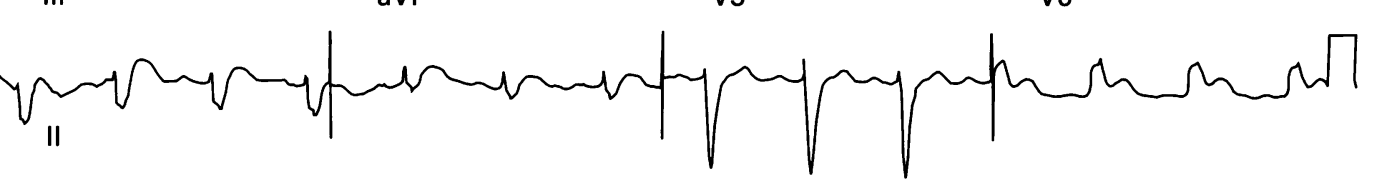

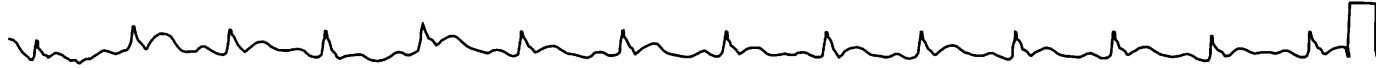

Figure $2 A$ subsequent ECG provides conclusive evidence of evolving AMI. There is now more marked ST segment elevation in leads II, III, and aVF as well as in leads V5 and V6. The ST segment and T wave changes in the lateral leads are concordant with the QRS complex.

chest pain accompanied by nausea, sweating, and shortness of breath. He had a history of diabetes and a previous myocardial infarction. A 12 lead ECG (fig 3) revealed sinus rhythm with LBBB. An ECG from the patient's medical records (fig 4) showed that the LBBB was pre-existing. Initially the ECG in fig 3 was considered remarkable only to the extent that it demonstrated sinus rhythm and LBBB. Once it was compared with the ECG in fig 4, it then became apparent that the patient may well be experiencing an acute anterior lateral myocardial infarction. This patient underwent emergency angiography with an angioplasty to a proximal lesion in the left anterior descending artery. AMI was confirmed by cardiac enzyme analysis.

\section{Discussion}

The accident and emergency doctor is often the first clinician to assess a patient with chest pain, interpret the ECG, and make an early therapeutic decision concerning reperfusion treatment. The electrocardiographic abnor- malities associated with acute ischaemic heart disease may be masked by LBBB. The ability to correctly interpret the ECG with the LBBB may influence the immediate management as well as the outcome. ${ }^{1-6}$ It is oft quoted that the electrocardiographic diagnosis of AMI is impossible in the presence of LBBB. Others state that the diagnosis of AMI is often straight forward and "disarmingly easy".

Patients with LBBB and AMI have a high mortality and morbidity. These patients should be rapidly and aggressively treated in the accident and emergency department-provided with thrombolytic treatment when appropriate. In patients with $A M I$, both the pre-existing LBBB and new onset LBBB are clinical markers for a significantly worse prognosis in terms of higher mortality, lower left ventricular ejection fraction, and increased incidence of cardiovascular complications. ${ }^{5-12}$ Despite this increased risk of poor outcomes, patients with LBBB frequently do not receive thrombolytic agents. ${ }^{13}{ }^{14}$ These patients show significant

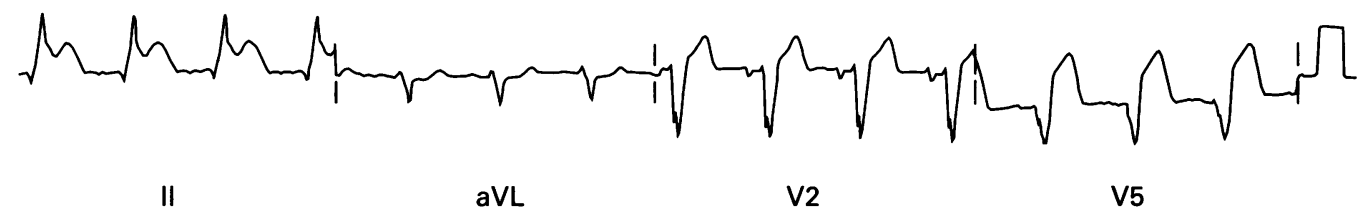

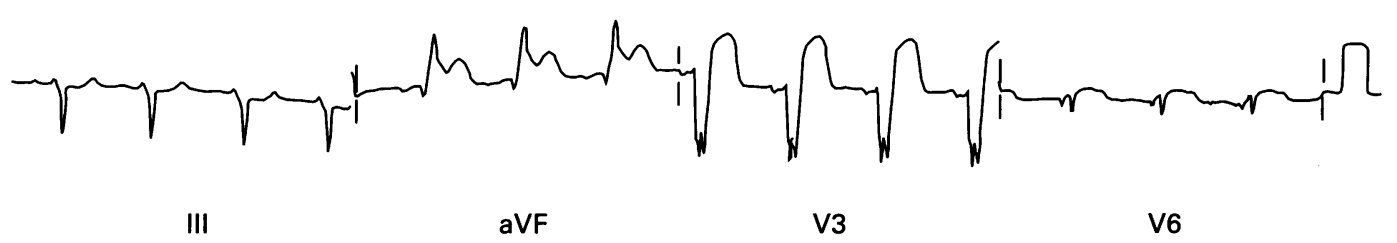

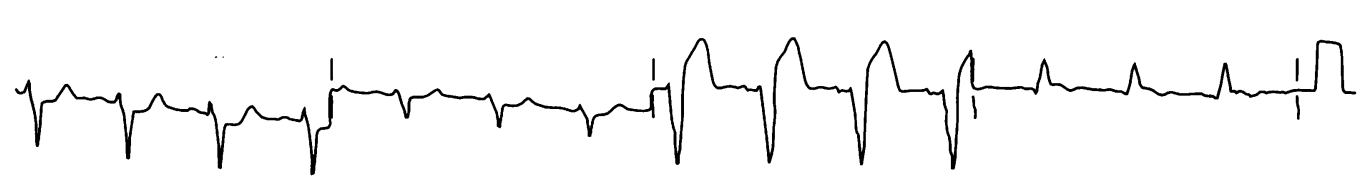

Figure 3 Close inspection of this ECG reveals $S T$ changes that are inconsistent with those expected with LBBB. Obvious $S T$ segment elevation is noted in leads I, aVL, and V6 suggesting acute infarction as the ST segments in these leads should be discordant with the QRS complex. Furthermore, examination of the height and contour of the ST segment elevation in leads V1-V4 compared with the previous baseline ECG (fig 5) indicated a new injury pattern consistent with anterior wall AMI. In summary this patient has the electrocardiographic changes of an anterolateral myocardial infarction. 

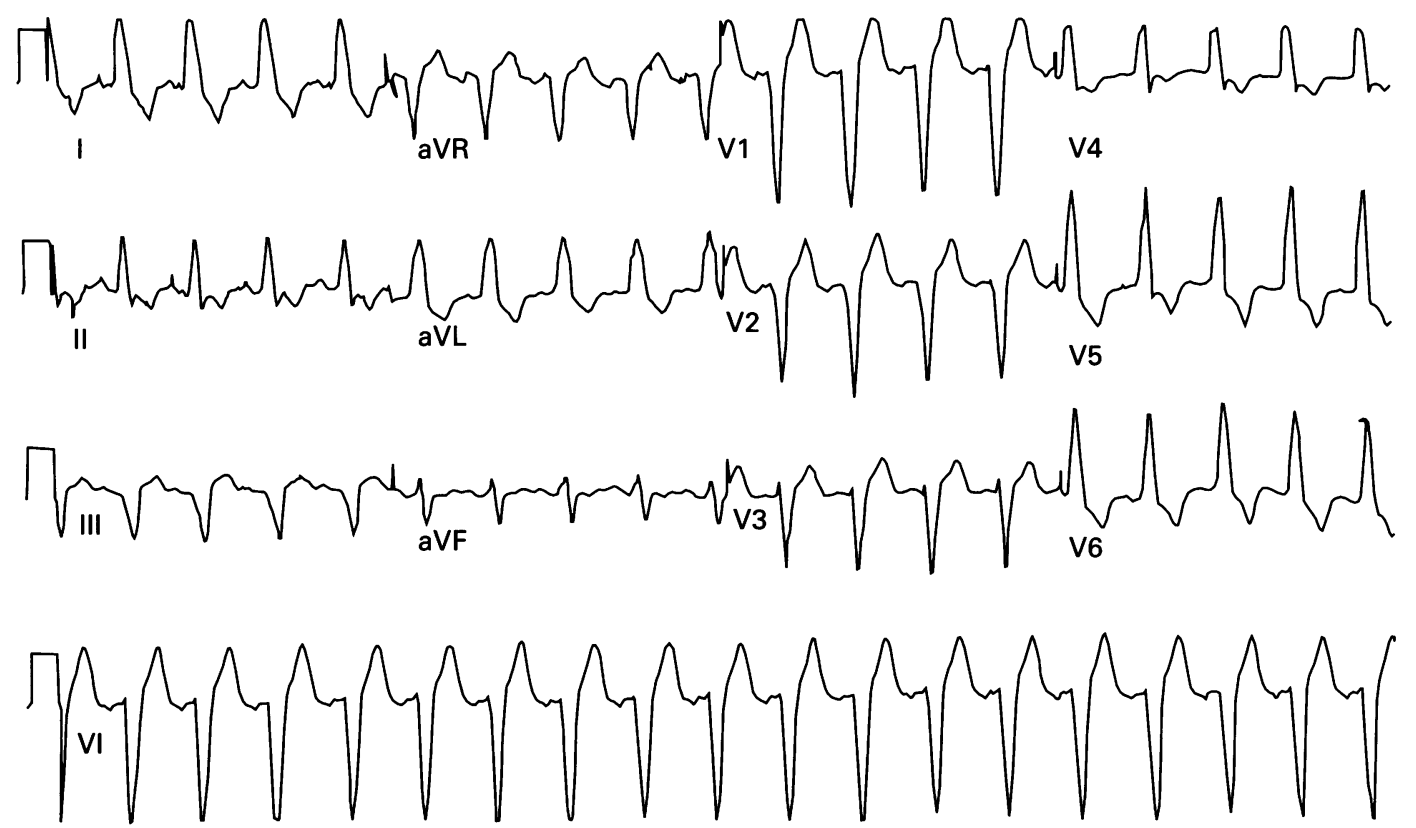

Figure 4 The baseline ECG of the patient in fig 4 demonstrates sinus rhythm and uncomplicated LBBB. The "normal" discordant nature of the $S T$ segments with reference to the $Q R S$ complexes are well illustrated.

benefits when treated with thrombolytic treatment. ${ }^{15}$

Doctors in the accident and emergency department should find the following strategies helpful when determining whether a patient with an ECG demonstrating LBBB also has changes compatible with AMI. Firstly, a clear understanding of the wave forms associated with the abnormal ventricular conduction found in LBBB is necessary, thus providing the physician with the ability to make the early diagnosis of AMI when the abnormalities are not the expected morphology. ${ }^{76-18}$ This approach was useful as seen in case 1 ; in case 2 , the initial clinician was not familiar with the appropriate $S T$ segment/T wave changes for the LBBB and therefore incorrectly interpreted the ECG. Secondly, the performance of serial ECGs in patients with LBBB may reveal evolving changes and assist in the evaluation of patients with suspected AMI, as seen in case 1 . Thirdly, a comparison of a recent ECG with an old study may provide invaluable insights into
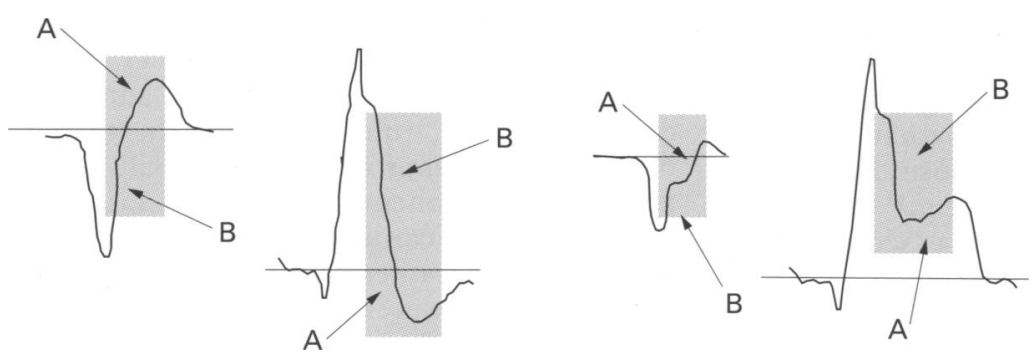

Figure 5 The anticipated or expected $S T$ segment changes in the patient with uncomplicated $L B B B$ are in the opposite direction to the $Q R S$ complex (termed discordance)-meaning any complex where the QRS is positive it is normally associated with a ST segment which is negative and vice versa. As a consequence, leads with either $Q S$ or $r S$ complexes (predominantly negative) may have markedly elevated $S T$ segments and vaulting $T$ waves mimicking AMI and leads with a large monophasic $R$ wave (primarily positive) will demonstrate ST segment depression. In these examples, both appropriate and inappropriate $S T$ segment/T wave and $Q R S$ complex relationships are demonstrated. In each example, " $A$ " refers to the initial ST segment/T wave while " $B$ " refers to the predominant, terminal portion of the $Q R S$ complex. In the normal setting for a $L B B B$ pattern, both " $A$ " and " $B$ " should be on opposite sides of the isoelectric baseline-that is, the discordant relationship. The two examples on the left reveal appropriate discordant relationships while the two right examples demonstrate concordant relationships that are suggestive but not diagnostic of acute coronary ischaemia. which changes are new and which are not, as seen in case 2 . In practice, however, old ECGs are often not immediately available; further, obtaining serial ECGs over a short period of time is not a practice that is widespread in accident and emergency departments in the UK. Accident and emergency doctors may therefore have to rely upon a single ECG or at best a series of two recordings on which to base the management decision. These limitations mitigate against early diagnosis. It is important therefore that accident and emergency clinicians are fully conversant with the normal electrocardiographic pattern of uncomplicated LBBB and therefore are in a position to recognise any unexpected changes. ${ }^{76-18}$

In a patient with uncomplicated $\mathrm{LBBB},{ }^{76}$ the 12 lead ECG records the abnormal ventricular activation as it moves from right to left, producing a broad, mainly negative QS or rS complex in the lead V1. In lead V6, a positive, monophasic $\mathrm{R}$ wave is noted; similar patterns are frequently found in lead I and aVl. Poor $\mathbf{R}$ wave progression or QS complexes are noted in the right to mid-precordial leads, rarely extending beyond leads V4 and V5. QS complexes may also be encountered in leads III and aVF. The anticipated or expected ST changes are in the opposite direction to the QRS complex (termed discordance), meaning any complex where the QRS is positive it is normally associated with an ST segment which is negative and vice versa (fig 5). As a consequence, leads with either $\mathrm{QS}$ or $\mathrm{rS}$ complexes (negative) may have markedly elevated ST segments and vaulting $T$ waves mimicking AMI, ${ }^{16-20}$ and leads with a large monophasic $\mathrm{T}$ wave demonstrate ST segment depression.

This discordant relationship is to be expected though it is often not reported in the descriptions of the $\mathrm{LBBB}$, resulting in the uninformed doctor making an incorrect diagnosis of an AMI and prescribing thrombolytic 


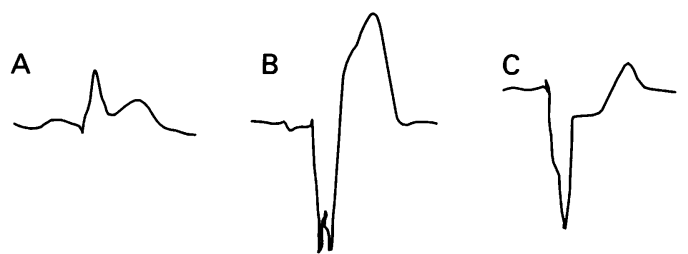

Figure 6 The electrocardiographic criteria according to Sgarbossa et al ${ }^{15}$ suggestive - not diagnostic of - $A M I$ include the following: $(A) S T$ segment elevation $>1 \mathrm{mV}$ that is concordant with the QRS complex; (B) ST segment elevation $>5 \mathrm{mV}$ that is discordant with the QRS complex; and $(C) S T$ segment depression $>1 \mathrm{mV}$ in leads V1, V2, or V3.

treatment inappropriately. ${ }^{21}$ One recent out-ofhospital study of patients with chest pain revealed that $51 \%$ of such patients with ST elevation of more than $1 \mathrm{~mm}$ in two contiguous leads were shown not to have had a myocardial infarction during their subsequent hospital stay. ${ }^{22}$ Left bundle branch was the second most frequently encountered electrocardiographic pattern responsible for this type of non-AMI ST elevation. The ST segment/T wave abnormalities encountered with LBBB reportedly make this entity the most frequently misinterpreted pseudoinfarction pattern in practice today. ${ }^{20}$ The electrocardiographic features of uncomplicated LBBB described above are demonstrated in fig 4. Loss of this normal QRS complex and ST segment discordance in patients with LBBB may suggest an acute ischaemic event. Close inspection of the ECG in patients with chest pain and LBBB should primarily be concerned with: (1) confirmation of LBBB and (2) evidence indicating the loss of discordance and/or the appearance of inappropriate discordance.

Sgarbossa et al developed a clinical prediction rule to assist in the electrocardiographic diagnosis of AMI in the setting of LBBB using specific findings. ${ }^{13}$ They analysed the numerous electrocardiographic abnormalities previously reported to be suspicious or diagnostic for AMI in patients with LBBB and identified three criteria suggestive of acute infarction. This rule, developed from 131 patients with LBBB and enzymatically proved AMI who were enrolled in the GUSTO-1 trial, states that three specific electrocardiographic criteria are independent predictors of AMI. The electrocardiographic criteria suggesting a diagnosis of AMI, ranked with a scoring system based on the probability of such a diagnosis, include:

(1) ST segment elevation $>1 \mathrm{mV}$ that is concordant with the QRS complex (score of 5, fig $6 \mathrm{~A})$.

(2) ST segment depression $>1 \mathrm{mV}$ in leads $\mathrm{V} 1$, V2, or V3 (score of 3, fig 6C).

(3) ST segment elevation $>5 \mathrm{mV}$ that is discordant with the QRS complex (score of 2, fig 6B).

A total score $>3$ (summed from all abnormal leads on the ECG) suggests that the patient is likely to be experiencing an AMI based on the electrocardiographic criteria. In patients with a score totalling $<3$, the electrocardiographic diagnosis is less convincing, requiring additional evaluation. This clinical prediction instrument supports the contention that a detailed knowledge of the anticipated ST segment $T$ wave changes associated LBBB is mandatory. Such an understanding of the ECG with LBBB allows the clinician to recognise the unanticipated changes that may be suspicious for AMI

Recent literature has suggested that the above clinical prediction rule is less useful than reported by the above authors in the real time evaluation of the adult chest pain patient with LBBB. $^{23}$ This review, which applied the Sgarbossa $e t a^{13}$ criteria to patients with chest pain and LBBB in the emergency department of a North American hospital, found much less promising results. In this application, the sensitivity for the electrocardiographic diagnosis of AMI was quite low; furthermore, the interobserver reliability among emergency physicians was poor. None the less, even if the clinical prediction rule is found to be less useful in the objective evaluation of the ECG in the patient with LBBB, the Sgarbossa et al report has merit ${ }^{13}$; it has forced the clinician to review the ECG in detail and cast some degree of doubt on the widely taught belief that the ECG is significantly invalidated in the search for AMI in the patient with LBBB.

\section{Conclusion}

The electrocardiographic diagnosis of AMI in the setting of LBBB is difficult but possible. The abilities of the accident and emergency doctor to correctly interpret the electrocardiographic findings for such complicated patients may directly affect the management. Several strategies are available to the accident and emergency clinician to help in this endeavour such as comparison with old ECGs and examination of serial ECGs. However, the most helpful strategy is the sound understanding of the anticipated ST segment changes resulting from LBBB and then the subsequent recognition of unexpected or pathological electrocardiographic changes. These strategies may be supplemented by the clinical decision rule developed by Sgarbossa et al. ${ }^{13}$ Using a knowledge of the anticipated electrocardiographic changes of LBBB supported by the criteria proposed by Sgarbossa et al, ${ }^{13}$ the physician's approach in the early phase of care can then move form a "non-diagnostic" ECG with a "rule-out" myocardial infarction admission diagnosis to a "rule-in" approach, thereby offering more appropriate, potentially more aggressive treatment in timely fashionincluding early cardiology consultation with urgent cardiac catheterisation. The physician must realise, however, that these ST segment changes are only suggestions of AMI in patients with complicated ECGs; by themselves, they are not diagnostic of AMI. Therapeutic decisions must be made with this caveat in mind.

Conflict of interest: none. Funding: none.

Muller DW, Topol EJ. Selection of patients with acute myocardial infarction for thrombolytic therapy. Ann Intern Med 1990;113:949-60.

2 Kleinman NS, White HD, Ohman EM, et al. Mortality within 24 hours of thrombolysis for myocardial infarction the importance of early reperfusion. Circulation 1994;90 2658-65. 
3 Lee TH, Weisberg MC, Brand DA, et al. Candidates for thrombolysis among emergency room patients with acute chest pain: potential true and false-positive rates. $A n n$

4 Bren GB, Wasserman AG, Ross AM. Electrocardiogram in patients undergoing thrombolysis for myocardial infarction. Circulation 1987;76(suppl II): 18-24

5 Fibrinolytic Therapy Trialists (FTT) Collaborative Group. Indications for fibrinolytic therapy in suspected acute myoand major morbidity results from all randomized trials of more than 1000 patients. Lancet 1994;343:311-22.

6 The GUSTO Investigators. An international randomized trial comparing four thrombolytic strategies for acute myocardial infarction. $N$ Engl f Med 1993;329:673-82.

7 Marriott HJI. Myocardial infarction. In: Marriott HJL, ed Practical electrocardiograph. 8th Ed. Baltimore: Williams and Wilkins, 1988: 419-50.

$8 \mathrm{Col} \mathrm{JJ}$, Eiknberg SL. The incidence of mortality of intraventricular conduction defects in acute myocardial infarction. Am $\mathcal{F}$ Cardiol 1972;29:344-50.

9 Hindman MC, Wagner GS, JaRo M, et al. The clinical significance of bundle branch block complication acute myocardial infarction. 1. Clinical characteristics, hospital mortality, and one-year follow-up. Circulation 1978;58:679-88

10 Opolski G, Kraska T, Ostrzycki A, et al. The effect of infarct size on atrioventricular and intraventricular conduction disturbances in acute myocardial infarction. Int $\mathcal{F}$ Cardio 1986;10:141-7.

11 Hamdy RI, Weissman RH, Prakash MN, et al. Left bundle branch block: a predictor of poor left ventricular function in coronary heart disease. Am Heart 7 1983;106:471-7.

12 Freedman RA, Alderman EL, Sheffield LT, et al. Bundle branch block in patient with chronic coronary artery disease: angiographic correlates and prognostic significance. $₹$ Am Coll Cardiol 1987;10:73-80.

13 Sgarbossa EB, Pinski SL, Barbagelata A, et al. Electrocardiographic diagnosis of evolving acute myocardial infarction in the presence of left bundle branch block. $N$ Engl $\mathcal{F}$ Med 1996;334:481-7.
14 Rogers WJ, Bowlby LI, Chandra NC, et al. Treatment of myocardial infarction in the United States (1990 to 1993): observations from the National Registry of Myocardial Infarction. Circulation 1994;90:2103-14.

15 ISIS-2 (Second International Study of Infarct Survival) Collaborative Group. Randomized trial of intravenous streptokinase, oral aspirin, both, or neither amount 17,187 cases of suspected acute myocardial infarction: ISIS-2. Lancet 1988;ii:349-60.

16 Aufderheide TP, Brady WJ. Electrocardiography in the patient with myocardial ischaemic or infarction. In: Gibler patient with myocardial ischaemic or infarction. In: Gibler Louis: Mosby, 1994: 169-216.

17 Hands ME, Cook EF, Stone PH, et al. Electrocardiographic diagnosis of myocardial infarction in the presence of complete left bundle branch block. Am Heart $\mathcal{F}$ 1988;116:23 31

18 Kuhn M. ECG diagnosis of acute myocardial infarction in patients with bundle branch block. Ann Emerg Med 1988;17:633-9.

19 Fesmire FM. ECG diagnosis of acute myocardial infarction in the presence of left bundle branch block in patients undergoing continuous ECG monitoring. Ann Emerg Med 1995;26:69-82.

20 Golderger AL. Myocardial infarction: electrocardiographic differential diagnosis. 4th Ed. St Louis: Mosby, 1991: 100-26.

21 Ohman EM, Sigmon KN, Califf RM. Is diagnostic certainty essential for the use of thrombolytic therapy during acute myocardial infarction in the 1990s? Circulation 1990;82 1073-5.

22 Otto LA, Aufderheide TP. Evaluation of ST segment elevation criteria for the prehospital electrocardiographic diagnosis of acute myocardial infarction. Ann Emerg Med 1994;23:17-24.

23 Shapiro NI, Fisher J, Zimmer GD, et al. Validation of electrocardiographic criteria for diagnosing acute myocardial infarction in the presence of left bundle branch block. Acad Emerg Med 1998;5:508 (abstract).

\section{Annual Scientific Meeting of the Faculty of Accident and Emergency Medicine}

\section{3-4 December 1999, Royal College of Physicians of London}

\section{Call for abstracts}

Forms are available from the Faculty of Accident and Emergency Medicine, 35-43 Lincoln's Inn Fields, London WC2A 3PN (tel: +44 (0) 171405 7071, fax: +44 (0) 1714050318 , e-mail: FAEM@compuserve.com)

Abstract forms must be received by 6 August 1999

Authors will receive notification of acceptance/rejection 